\title{
Technical note: Flow cytometric identification of bovine milk neutrophils and simultaneous quantification of their viability
}

\author{
S. Piepers, ${ }^{* 1}$ S. De Vliegher, ${ }^{\star}$ K. Demeyere,† B. N. Lambrecht, $\neq$ A. de Kruif, ${ }^{\star}$ E. Meyer, † and G. Opsomer ${ }^{\star}$ \\ *Department of Reproduction, Obstetrics, and Herd Health, Faculty of Veterinary Medicine, Ghent University, 9820 Merelbeke, Belgium \\ †Department of Pharmacology, Toxicology, Biochemistry, and Organ Physiology, Laboratory of Biochemistry, Faculty of Veterinary Medicine, \\ Ghent University, 9820 Merelbeke, Belgium \\ ‡Department of Internal Medicine subdivision Pneumology, Faculty of Medicine and Health Sciences, Ghent University, 9000 Ghent, Belgium
}

\begin{abstract}
The aim of the present study was to develop a flow cytometric procedure for the quantification of the proportion of viable, apoptotic, and necrotic polymorphonuclear neutrophilic leukocytes (PMNL) isolated from both low- and high-somatic-cell-count quarter milk samples. Milk PMNL were differentiated from other cells by indirect fluorescent labeling using a primary anti-bovine granulocyte monoclonal antibody (CH138A) and an Alexa 647-labeled secondary antibody. Polymorphonuclear neutrophilic leukocytes were identified flow cytometrically based on their cytoplasmic granularity and CH138A-positivity. Additional labeling with annexinV-fluorescein isothiocyanate and propidium iodide was used to determine milk PMNL viability. Thirty milk samples were run in parallel to assess the repeatability of the immunoassay and 6 repeated measurements per sample were performed to assess the instrument stability. Fluorescence microscopic verification of the CH138A staining pattern showed both a high sensitivity $(90.9 \%)$ and specificity (92.3\%). The combination of the side-scatter properties of granulated PMNL and CH138A-Alexa 647 positivity allows the distinction of labeled PMNL from other milk cells and particles that may bind nonspecifically, and from autofluorescent particles present in milk. Quantification of the proportion of PMNL and viable, apoptotic, and necrotic subpopulations in parallel samples gave repeatable results with concordance correlation coefficients varying between 0.93 and 0.99 . The average coefficient of variation for repeated measurements in identical samples ranged between 4.2 and $9.7 \%$. In conclusion, this is the first flow cytometric method suited for the simultaneous quantification of viable, apoptotic, and necrotic bovine milk PMNL in a straightforward manner.
\end{abstract}

Key words: bovine, flow cytometry, milk, neutrophil

Received May 26, 2008.

Accepted October 13, 2008

${ }^{1}$ Corresponding author: Sofie.Piepers@UGent.be
Evidence suggests that the first-line immune defense of the bovine mammary gland is mainly determined by the number and function of the resident and incoming PMNL (Rainard and Riollet, 2006). Variations in the viability of milk PMNL are therefore believed to at least partly explain differences among dairy cows and quarters in the resolution of existing intramammary infections and in the resistance to new ones (Mehrzad et al., 2004; Sládek and Ryšanek, 2005). After all, apoptosis of bovine milk PMNL substantially affects their functionality (Van Oostveldt et al., 2002; Mehrzad et al., 2004). Various flow cytometric differential leukocyte methods are available for the analysis of high-SCC milk as well as for the most technically challenging analysis of low-SCC milk (Miller et al., 1993; Dosogne et al., 2003; Koess and Hamann, 2008). Flow cytometry allows rapid and automatic processing of a substantial number of samples and of a large number of cells within a sample at a time (van Eeden et al., 1999) and is therefore less laborious than conventional cytology and fluorescence microscopy (van Eeden et al., 1999; Feng and Zheng, 2005). None of the abovementioned methods based on flow cytometry has been developed to identify PMNL and to simultaneously quantify their viability. Since bench-top instrument features and analytical software have progressed considerably during the last 5 yr, the aim of the present study was to develop a simple flow cytometric method able to identify milk PMNL and to simultaneously quantify viable, apoptotic, and necrotic subpopulations from both low- and high-SCC milk samples, that can be employed in field studies.

Thirty quarter foremilk samples from 15 randomly selected, clinically healthy primiparous $(\mathrm{n}=6)$ and multiparous $(n=9)$ cows were collected at the research dairy farm of the Faculty of Veterinary Medicine, Ghent University, Belgium. Milk samples $(50 \mathrm{~mL})$ for the quantification of PMNL viability and the determination of the SCC (30 mL, Fossomatic 5000, Foss Electric, Hillerød, Denmark) were aseptically collected in the morning after the first streams of milk were discarded. All milk samples were collected within $4 \mathrm{~d}$ after calving, because this is generally accepted as the critical time 
to study the association between innate immunity and mastitis (Rainard and Riollet, 2006). Eight samples had a SCC $\leq 200,000$ cells $/ \mathrm{mL}$ (geometric mean SCC of 57,000 cells/mL, ranging from 22,000 to 159,000 cells/ $\mathrm{mL}), 7$ samples between 201,000 and 500,000 cells $/ \mathrm{mL}$ (geometric mean SCC of 349,000 cells $/ \mathrm{mL}$, ranging from 237,000 to 499,000 cells/mL), 9 samples between 501,000 and 1,000,000 cells/mL (geometric mean SCC of 732,000 cells $/ \mathrm{mL}$, ranging from 638,000 to 841,000 cells $/ \mathrm{mL}$ ), and 6 samples $\geq 1,000,000$ cells $/ \mathrm{mL}$ (geometric mean SCC of $2,141,000$ cells $/ \mathrm{mL}$, ranging from $1,179,000$ to $9,999,000$ cells $/ \mathrm{mL}$ ).

Isolation of milk leukocytes was based on a previously published method (Mehrzad et al., 2002). The initial volume of quarter foremilk was divided equally between two 50-mL Falcon tubes, diluted 50\% (vol/vol) with cold PBS, and centrifuged at $300 \times g$ for $15 \mathrm{~min}$. All centrifugation steps were carried out at $4^{\circ} \mathrm{C}$. After carefully removing the cream layer, the pellet was suspended into $10 \mathrm{~mL}$ of PBS, centrifuged at $200 \times g$ for $10 \mathrm{~min}$, and washed 2 more times. The final milk cell pellet was suspended in $500 \mu \mathrm{L}$ of RPMI 1640 (Gibco Brl., Scotland, UK) supplemented with 1\% BSA (Merck KGaA, Darmstadt, Germany), hereafter referred to as RPMI-BSA. After counting the milk cells with a cell counter (Coulter Counter, Analis, Suarlée, Belgium), the cell pellet was adjusted to a total of $2 \times 10^{5}$ cells in each flow cytometer tube, and again centrifuged at $200 \times g$ for $10 \mathrm{~min}$. The 2 identical samples A and B were subsequently treated in parallel. The resulting 60 samples were used to assess the repeatability of the immunoassay.

To identify PMNL, the milk cell pellets were incubated for $30 \mathrm{~min}$ in a warm water bath at $37^{\circ} \mathrm{C}$ with $100 \mu \mathrm{L}$ of primary mouse IgM monoclonal antibody (mAb) CH138A diluted in RPMI-BSA to a final concentration of $10 \mu \mathrm{g} / \mathrm{mL}$, according to the manufacturer's directions (VMRD Inc., Pullman, WA). After incubation, the cells were centrifuged at $200 \times g$ for $10 \mathrm{~min}$ and washed 3 more times with $250 \mu \mathrm{L}$ of RPMI-BSA. Subsequently, the remaining cell pellet was incubated with $100 \mu \mathrm{L}$ of Alexa 647-labeled goat anti-mouse IgM antibody (Ab) (Molecular Probes, Leiden, the Netherlands) diluted in RPMIBSA to a final concentration of $4.8 \mu \mathrm{g} / \mathrm{mL}$. After $30 \mathrm{~min}, 250 \mu \mathrm{L}$ of RPMI-BSA was added, the cell suspension was centrifuged at $200 \times g$ for $10 \mathrm{~min}$, and 2 more wash steps and centrifugation steps were performed with $250 \mu \mathrm{L}$ of RPMI-BSA. On 2 milk samples, an additional immunolabeling with the $\mathrm{mAb}$ anti-bovine CD14 (Clone CAM36A, VMRD Inc.) and the phycoerythrin-labeled secondary anti-mouse $\operatorname{IgG}$ Ab (Sigma-Aldrich, Bornem, Belgium) was conducted as mentioned above to detect mononuclear cells, in particular macrophages (Riollet et al., 2001).

The proportion of viable, apoptotic, and necrotic milk PMNL was determined by carrying out double staining with annexin-V-fluorescein isothiocyanate (FITC; Roche Molecular Biochemicals, Indianapolis, IN) and propidium iodide (PI; Sigma-Aldrich; Vermes et al., 1995). One milliliter of a $\mathrm{Ca}^{++}$-rich incubation buffer (10 $\mathrm{m} M$ HEPES, $140 \mathrm{~m} M \mathrm{NaCl}, 5 \mathrm{~m} M \mathrm{CaCl}_{2}$, pH 7.4) was prepared and $20 \mu \mathrm{L}$ of annexin-V-FITC and $20 \mu \mathrm{L}$ of PI (final concentration of $1 \mu \mathrm{g} / \mathrm{mL}$ ) were added. One hundred microliters of this mixture was added to the CH138A-labeled milk cell pellet, and incubated for 10 min in the dark. All data were acquired and processed using FACSDiva Software (Becton, Dickinson and Company, Franklin Lakes, NJ).

The staining pattern of the anti-bovine granulocyte marker CH138A was confirmed by fluorescence microscopy. Isolated milk cells were first incubated with the primary $\mathrm{mAb} \mathrm{CH} 138 \mathrm{~A}$ as aforementioned, followed by incubation for $30 \mathrm{~min}$ at $4^{\circ} \mathrm{C}$ with $100 \mu \mathrm{L}$ of FITClabeled secondary $\mathrm{Ab}$ rabbit anti-mouse $\mathrm{IgM}$ (final concentration of $4 \mu \mathrm{g} / \mathrm{mL}$ ) to visualize the CH138A binding, and with $50 \mu \mathrm{L}$ of PI (final concentration of $50 \mu \mathrm{g} / \mathrm{mL}$ ) to demonstrate the multilobulated morphology, typical for the PMNL nucleus. The sensitivity was defined as the proportion of CH138A-positive PMNL as characterized by a multilobulated nucleus, whereas the specificity was defined as the proportion of CH138Anegative mononuclear cells (non-PMNL). Additionally, flow cytometric cell sorting of CH138A-labeled milk cells with a high granularity was performed (FACSAria II, BD Biosciences, San Jose, CA). The sorted cell population was stained (Hemacolor Solutions, Merck) after preparing the slides by cytocentrifugation (Shandon, Southern Products Ltd., Runcorn, UK) and subsequently analyzed by light microscopy (BX-UCB, Olympus, Center Valley, PA).

To evaluate the assays' repeatability, the concordance correlation coefficients with the lower bound $95 \%$ confidence interval (CI) of 2 parallel samples A and B from each of the 30 quarter foremilk samples were calculated for the proportion of PMNL and the proportion of viable, apoptotic, and necrotic subpopulations (Dohoo et al., 2003). The stability of the flow cytometric measurement of percentage PMNL, percentage viable PMNL, percentage apoptotic PMNL, and percentage necrotic PMNL was tested by performing 6 repeated measurements on one test sample (Dohoo et al., 2003). The sample coefficient of variation $\left(\mathbf{C V}_{\text {sample }}\right)$ based on 6 repeated measurements on one test sample was defined as $\mathrm{CV}_{\text {sample }}=\sigma_{\text {sample }} / \mu_{\text {sample }}$ where $\sigma_{\text {sample }}=$ the standard deviation of the 6 repeated measurements per sample 
and $\mu_{\text {sample }}=$ the average of the 6 repeated measurements per sample. Subsequently, the average coefficient of variation $\left(\mathbf{C V}_{\text {average }}\right)$ was estimated as $\mathrm{CV}_{\text {average }}=$ $\left(\Sigma_{\mathrm{n}} \mathrm{CV}_{\text {sample }}\right) / \mathrm{n}$ with $\mathrm{n}=30$.

To explore the role of milk PMNL in the immunity of the mammary gland in field studies and clinical trials, processing of a considerable number of milk samples is needed. This is only possible when a precise and accurate method enabling automatic identification of milk PMNL and quantification of their viability in both lowand high-SCC milk is available. Milk cells were recently differentiated into low- and high-SCC samples using a flow cytometric template based on the separate immunolabeling with primary antibodies and with a common FITC-labeled secondary antibody (Koess and Hamann, 2008). The PMNL-region was defined with a mAb anti-bovine CD11b, mainly reacting with granulocytes. Within this template, viable and necrotic milk PMNL were distinguished using PI. Although this approach represents a progress with respect to the former flow cytometric protocols to study milk PMNL, no further distinction between the viable and apoptotic nonviable fractions could be made. This drawback was elegantly circumvented in our 3-color flow cytometric protocol. Using a double-laser flow cytometer with excitation wavelengths at $488 \mathrm{~nm}$ and $633 \mathrm{~nm}$ allowed us to add annexin-V-FITC having a minimal spectral overlap with PI and Alexa 647 and enabling the discrimination between (early) apoptotic and necrotic PMNL. Milk PMNL were differentiated from other milk cells based on their cytoplasmic granularity and mean fluorescence intensity following 2-step fluorescent immunolabeling with the primary anti-bovine granulocyte Ab CH138A and the secondary Ab coupled to the long-wavelength fluorescent probe Alexa 647. The selected primary mouse IgM Ab CH138A is the only commercial $\mathrm{mAb}$ that reacts specifically with membrane antigens expressed on the surface of bovine granulocytes (Davis et al., 1987), and has already been successfully applied in other studies (Mehrzad et al., 2001; Lamote et al., 2004). Fluorescence microscopic verification of the CH138A staining pattern showed both a high sensitivity and specificity with $90.9 \%$ of the PMNL being CH138A-positive, and 92.3\% of the other milk cells being CH138A-negative, respectively. Light microscopic analysis of the sorted CH138A-labeled cells with high granularity confirmed that those cells are indeed of PMNL morphology (Figure 1). No isotypic control was incorporated because the usefulness and interpretation of isotype antibodies as a quality control measure to determine the nonspecific background fluorescence is still debated (Keeney et al., 1998; O'Garmon and Thomas, 1999). Moreover, the high protein content of milk ensures that milk cells are adequately blocked. As secondary antibody, a goat

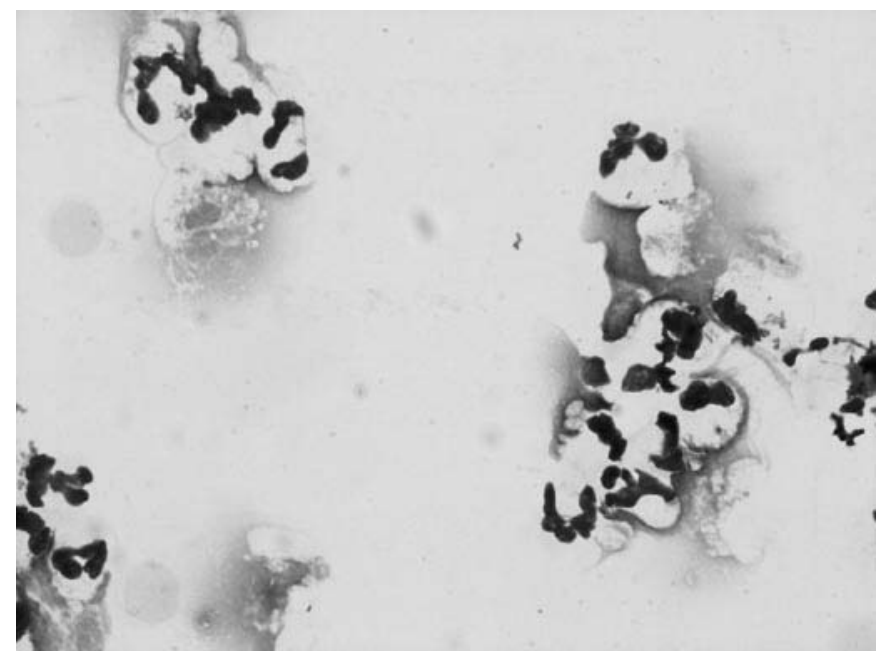

Figure 1. Light microscopic analysis of the CH138A-positive cell population with high granularity after cell sorting (magnification at $60 \times)$.

anti-mouse IgM protein specifically reacting with the primary Ab IgM heavy chains and labeled with the Alexa 647 dye was selected.

To illustrate the discrimination potential of the current flow cytometric method between milk PMNL and other milk cells, results of a representative high- and low-SCC quarter foremilk sample are depicted (Figure 2A-I; Figure 2A-II). Milk cells were first evaluated based on their FSC and SSC properties. Polymorphonuclear leukocytes are expected to be mainly located in the upper right part of the dot plot as previously determined using purified preparations of isolated milk PMNL (Mehrzad et al., 2002). They were identified using the SSC versus Alexa 647 dot-plot by drawing a gating region to enclose the PMNL based on cytoplasmic granularity and CH138A positivity (Figure 2B-I; Figure 2B-II). Back-gating the small population of events with moderate to high Alexa 647 fluorescence, but with low granularity on the FSC versus SSC plot showed that these cells were partly located in the cellular debris region. In the study of Davis et al. (1987) evaluating CH138A as a granulocyte marker for bovine blood leukocytes, nonspecific binding of the $\mathrm{mAb}$ was only occasionally observed, presumably because of a smaller number of interfering particles in blood compared with milk. Another small subpopulation of the nonspecific binding events represents macrophages as identified by a strong expression of CD14 using the mAb anti-bovine CD14 marker (Riollet et al., 2001; data not shown). Phagocytosing macrophages in milk can have a variable size and granularity which may interfere with the scatter pattern of milk PMNL (Koess and Hamann, 2008). However, only 3\% of the CD14- 
positive cells were identified within the PMNL-gate. Polymorphonuclear neutrophilic leukocytes can express CD14-receptors on their surface as well, which likely explains the observed moderate CD14-positive cells within the PMNL-gate (Paape et al., 1996). Besides the nonspecific binding, the isolated milk cells sometimes showed high matrix-dependent background fluorescence (Figure 3), corroborating observations from previous studies using fluorescence detection (Rivas et al., 2002; Pulgarín et al., 2005). Milk is a complex biological matrix containing high concentrations of milk proteins and lipids, including caseins, fat globules, as well as fat- and water-soluble vitamins, all exhibiting characteristic fluorescence emission upon excitation (Lakowicz, 1999). The analysis of milk samples collected between 1 and $4 \mathrm{~d}$ in milk is very relevant from
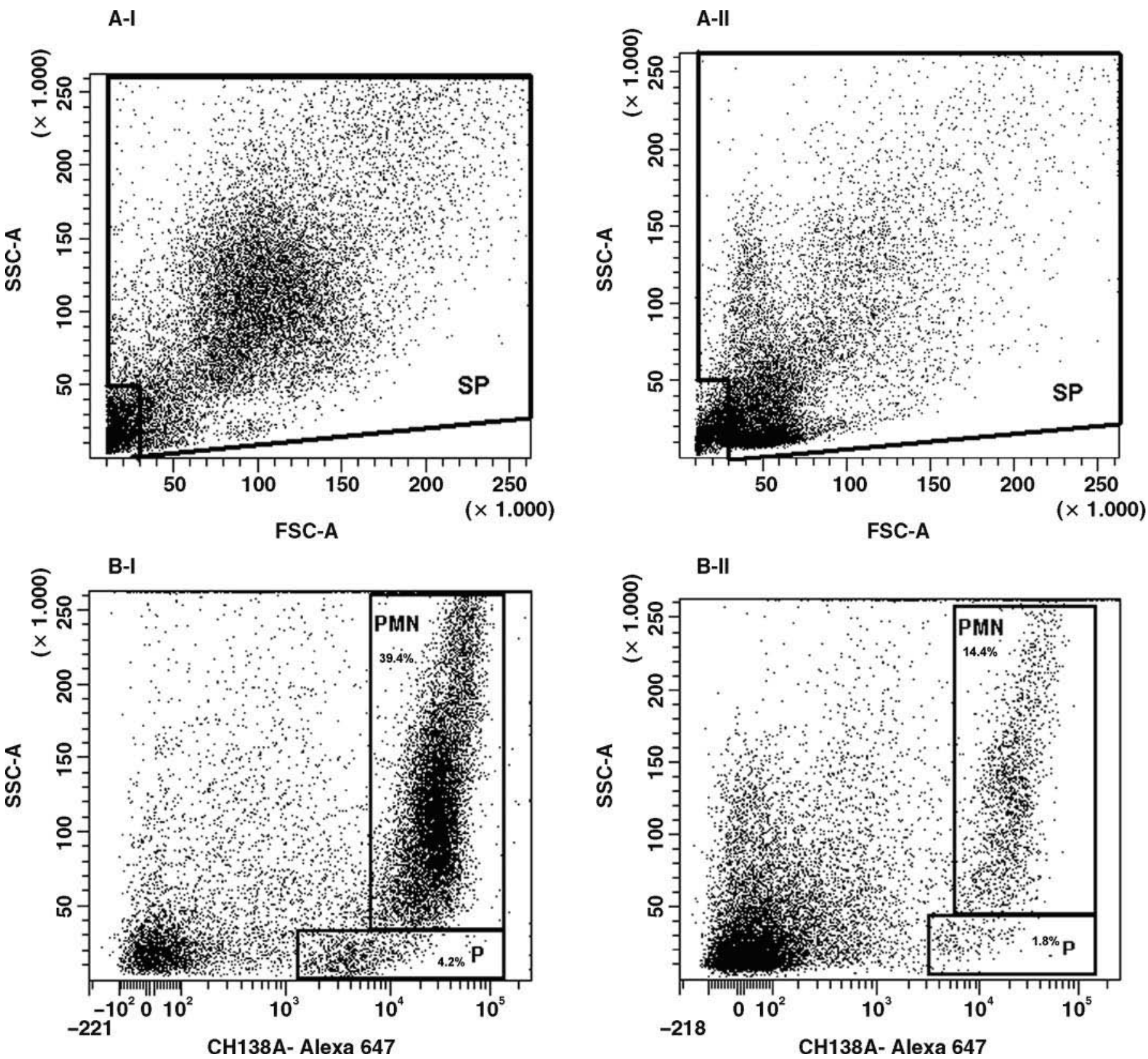

Figure 2. Flow cytometric identification of milk PMNL isolated from a representative (I) high-SCC $(\mathrm{SCC}=2,558,000$ cells $/ \mathrm{mL})$ and a representative (II) low-SCC quarter foremilk sample ( $\mathrm{SCC}=75,000$ cells $/ \mathrm{mL}$ ). Recordings of scatter and fluorescent properties were performed on 10,000 events gated in a standard population (SP) excluding most cell debris. Forward angle light scatter versus side angle light scatter (SSC) dot plot (A), and an SSC versus CH138A-Alexa 647 dot plot with PMNL = polymorphonuclear leukocytes and P = a subpopulation of nonspecifically bound milk cells and cellular debris (B). 


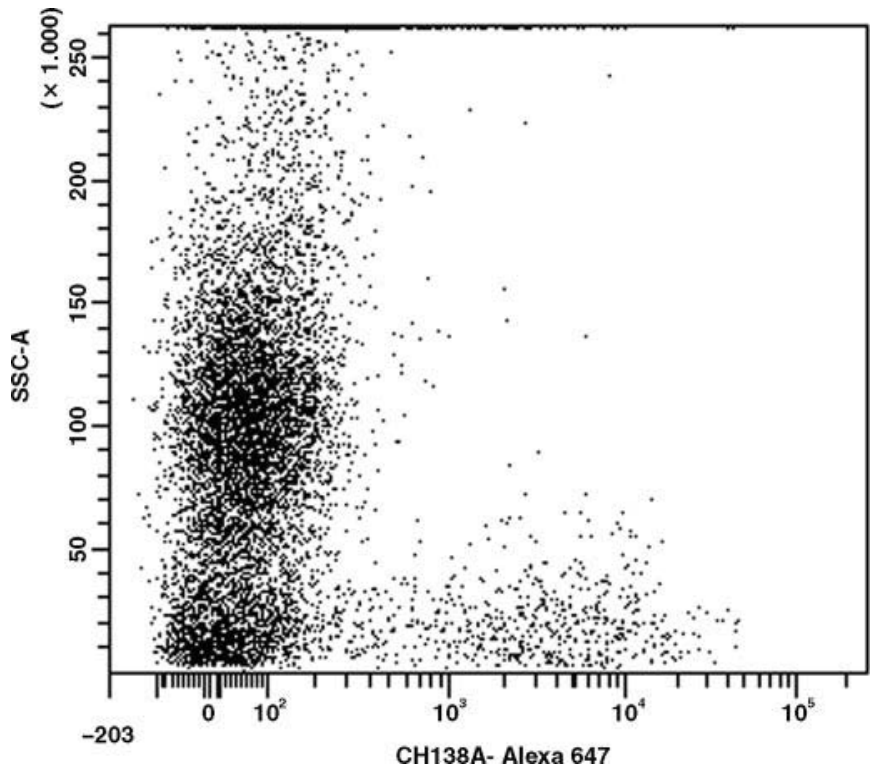

Figure 3. Representative flow cytometric SSC versus CH138AAlexa 647 dot plot of the background fluorescence in a milk sample with a high $\mathrm{SCC}(=2,558,000$ cells $/ \mathrm{mL})$.

a biological point of view, but is especially challenging because these samples are very rich in fat and proteins (Ontsouka et al., 2003). Nevertheless, labeled PMNL could systematically be differentiated from other milk cells as well as from autofluorescent particles present in milk based on their typical cytoplasmic granularity.

Staining cells simultaneously with annexin V-FITC and PI allowed the discrimination between intact milk PMNL (FITC ${ }^{-} / \mathrm{PI}^{-}$subpopulation), early apoptotic $\left(\mathrm{FITC}^{+} / \mathrm{PI}^{-}\right.$subpopulation) and late apoptotic or necrotic cells $\left(\mathrm{FITC}^{+} / \mathrm{PI}^{+}\right.$subpopulation; Figure 4). In the current study, the mean proportion of viable and apoptotic milk PMNL ranged between 10.5 and $85.9 \%$, and between 11.7 and $58.7 \%$, respectively. Apoptosis of milk PMNL is closely related to the PMNL functionality (Van Oostveldt et al., 2002; Mehrzad et al., 2004), and plays a key role in both the defense against invading pathogens (Boutet et al., 2004; Mehrzad et al., 2004) and the resolution of an inflammatory process (Van Oostveldt et al., 2002; Rainard and Riollet, 2006). The average proportion of necrotic milk PMNL was $25.4 \%$ (range $2.6-74.5 \%$ ), corresponding well with the results of Koess and Hamann (2008). Milk PMNL viability only decreased slightly and in a reproducible manner throughout the different washing and centrifugation steps, clearly excluding the possibility that the wide range in PMNL viability observed in the present study is caused by sample processing (data not shown). Moreover, data from different studies confirm that the mean proportion of viable milk PMNL ranges widely; that is, between 25.7 and $92.8 \%$ (Van Oostveldt et al., 2001; Mehrzad et al., 2002). As milk PMNL viability depends on the infection status (Boutet et al., 2004; Mehrzad et al., 2004), time of onset of the infection (Slàdek and Ryšanek, 2005), and parity and stage of lactation (Van Oostveldt et al., 2001; Mehrzad et al., 2002), comparison of our results with those from other studies should be done with caution.

The concordance correlation coefficients for the proportion of PMNL, and the proportion of viable, apoptotic, and necrotic subpopulations were estimated at 0.99 (lower bound 95\% CI: 0.98), 0.96 (lower bound 95\% CI: 0.94), 0.93 (lower bound 95\% CI: 0.88) and 0.99 (lower bound 95\% CI: 0.98), respectively. The values indicate an almost perfect repeatability for the proportion of PMNL and the necrotic subpopulation, and a high and substantial repeatability for the proportion of viable and apoptotic PMNL, respectively. The average $\mathrm{CV}$ for repeated measurements in identical test samples ranged between 4.2 and $9.7 \%$. Hence, a satisfactory repeatability for the 2-step immunofluorescent detection method and adequate instrument stability were obtained.

In conclusion, our method provides the possibility to flow cytometrically identify and quantify the viable, apoptotic, and necrotic bovine milk PMNL subpopulations. The unequivocal distinction of labeled PMNL from other milk cells and from autofluorescent particles present in milk was achieved by combining the SSC properties of granulated PMNL with the CH138A-Alexa

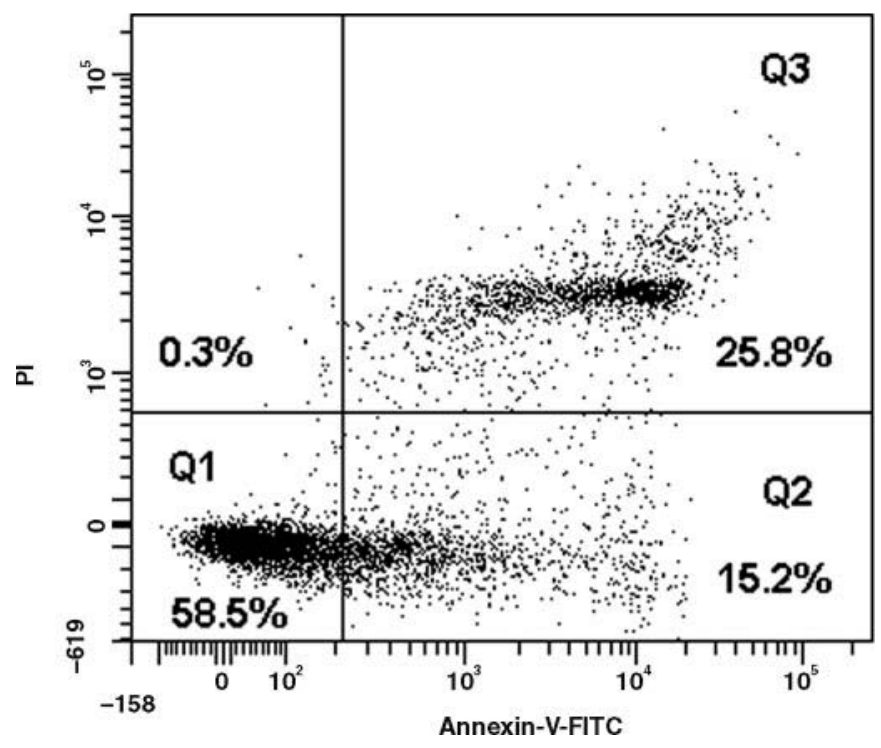

Figure 4. Representative flow cytometric annexin-V-fluorescein isothiocynate (FITC) versus propidium iodide (PI) dot plot showing the viable (Q1), apoptotic (Q2) and necrotic (Q3) CH138A-positive events in milk with a moderate SCC $(=391,000$ cells $/ \mathrm{mL})$. 
647 positivity. Although the preparative centrifugation and washing steps are relatively time-consuming, the presented protocol is easy to perform. Moreover, flow cytometry allows the rapid processing and objective judgment of a substantial number of samples and cells per sample at a time.

\section{ACKNOWLEDGMENTS}

This research was funded by a $\mathrm{PhD}$ grant to S. Piepers (no. 3422) by the Institute for the Promotion of Innovation through Science and Technology in Flanders (IWT Vlaanderen). The authors would like to thank the Milk Control Centre Flanders (Lier, Belgium) for the somatic cell count analyses and L. Baele (Hogeschool Gent, Ghent, Belgium), R. Schokkaert (KAHO Sint-Lieven Gent, Ghent, Belgium), and K. De Swarte (Department of Internal Medicine, Ghent University, Ghent, Belgium) for their excellent technical assistance.

\section{REFERENCES}

Boutet, P., D. Boulanger, L. Gillet, A. Vanderplasschen, R. Closset, F. Bureau, and P. Lekeux. 2004. Delayed neutrophil apoptosis in bovine subclinical mastitis. J. Dairy Sci. 87:4104-4114.

Davis, W. C., S. Marusic, H. A. Lewin, G. A. Splitter, L. E. Perryman, T. C. Mcguire, and J. S. Gorham. 1987. The development and analysis of species specific and cross reactive monoclonal antibodies to leukocyte differentiation antigens and antigens of the major histocompatibility complex for use in the study of the immune system in cattle and other species. Vet. Immunol. Immunopathol. 15:337-376.

Dohoo, I., M. Wayne, and H. Stryhn. 2003. Veterinary Epidemiologic Research. AVC Inc., Charlottetown, Prince Edward Island, Canada.

Dosogne, H., F. Vangroenweghe, J. Mehrzad, A. M. Massart-Leën, and C. Burvenich. 2003. Differential leukocyte count method for bovine low somatic cell count milk. J. Dairy Sci. 86:828-834.

Feng, W., and X. D. Zheng. 2005. Comparing techniques for detecting the number of somatic cells in raw milk. Eur. Food Res. Technol. 220:653-657.

Keeney, M., J. W. Gratama, I. H. Chin-Yee, and D. R. Sutherland. 1998. Isotype controls in the analysis of lymphocytes and CD34+ stem and progenitor cells by flow cytometry-Time to let go! Cytom. B 34:280-283.

Koess, C., and J. Hamann. 2008. Detection of mastitis in the bovine mammary gland by flow cytometry at early stages. J. Dairy Res. 75:225-232.

Lakowicz, J. R. 1999. Principles of Fluorescence Spectroscopy. 2nd ed. Kluwer Academic/Plenum, New York, NY.

Lamote, I., E. Meyer, L. Duchateau, and C. Burvenich. 2004. Influence of 17ß-estradiol, progesterone, and dexamethasone on diapedesis and viability of bovine blood polymorphonuclear leukocytes. J. Dairy Sci. 87:3340-3349.

Mehrzad, J., H. Dosogne, E. Meyer, R. Heyneman, and C. Burvenich. 2001. Respiratory burst activity of blood and milk neutrophils in dairy cows during different stages of lactation. J. Dairy Res. 68:399-415.

Mehrzad, J., L. Duchateau, and C. Burvenich. 2004. Viability of milk neutrophils and severity of bovine coliform mastitis. J. Dairy Sci. 87:4150-4162

Mehrzad, J., L. Duchateau, S. Pyörälä, and C. Burvenich. 2002. Blood and milk neutrophil chemiluminescence and viability in primiparous and pluriparous dairy cows during late pregnancy around parturition and early lactation. J. Dairy Sci. 85:32683276 .

Miller, R. H., M. J. Paape, R. Filep, and S. Link. 1993. Flow cytometric analysis of neutrophils in cows' milk. Am. J. Vet. Res. 54:19751979 .

O'Garmon, M. R. G., and J. Thomas. 1999. Isotype controls-Time to let go? Cytom. Part B 38:78-80.

Ontsouka, C. E., R. M. Bruckmaier, and J. W. Blum. 2003. Fractionized milk composition during removal of colostrums and mature milk. J. Dairy Sci. 6:2005-2011.

Paape, M. J., E. M. Lilius, P. A. Wiitanen, M. P. Kontio, and R. H. Miller. 1996. Intramammary defense against infections induced by Escherichia coli in cows. Am. J. Vet. Res. 57:477-482.

Pulgarín, J. A. M., A. A. Molina, and M. T. A. Pardo. 2005. Fluorescence characteristics of several whey samples subjected to different treatments and conditions. Anal. Chim. Acta 536:153158.

Rainard, P., and C. Riollet. 2006. Innate immunity of the bovine mammary gland. Vet. Res. 37:369-400.

Riollet, C., P. Rainard, and B. Poutrel. 2001. Cell subpopulations and cytokine expression in cow milk in response to chronic Staphylococcus aureus infection. J. Dairy Sci. 84:1077-1084.

Rivas, A. L., R. Tadevosyan, F. W. Quimby, T. Coksaygan, and D. H. Lein. 2002. Identification of subpopulations of bovine mammary gland phagocytes and evaluation of sensitivity and specificity of morphologic and functional indicators of bovine mastitis. Can. J. Vet. Res. 66:165-172.

Sládek, Z., and D. Ryšanek. 2005. Neutrophil apoptosis during the resolution of bovine mammary gland injury. Res. Vet. Sci. 70:4146.

van Eeden, S. F., M. E. Klut, B. A. M. Walker, and J. C. Hogg. 1999. The use of flow cytometry to measure neutrophil function. J Immunol. Methods 232:23-43.

Van Oostveldt, K., M. J. Paape, H. Dosogne, and C. Burvenich. 2002. Effect of apoptosis on phagocytosis, respiratory burst and CD18 adhesion receptor expression of bovine neutrophils. Domest. Anim. Endocrinol. 22:37-50.

Van Oostveldt, K., F. Vangroenweghe, H. Dosogne, and C. Burvenich. 2001. Apoptosis and necrosis of blood and milk polymorphonuclear leukocytes in early and midlactating healthy cows. Vet. Res. 32:617-622.

Vermes, I., C. Haanen, H. Stefferns-Nakken, and C. Reutelingsperger. 1995. A novel assay for apoptosis: Flow cytometric detection of phosphatidylserine expression on early apoptotic cells using fluorescein labelled Annexin-V. J. Immunol. Methods 184:39-51. 